\title{
A method for measuring relative in-plane diffusivity of thin and partially saturated porous media: an application to fuel cell gas diffusion layers
}

T. G. Trantera,c, P. Stogornyuk ${ }^{\mathrm{b}}$, J. T. Gostick ${ }^{\mathrm{b}, \mathrm{c}}$, A. D. Burns ${ }^{\mathrm{a}}$, W. F. Gale ${ }^{\mathrm{a}}$

a Centre for Integrated Energy Research, School of Chemical and Process Engineering, University of Leeds, Leeds, LS2 9JT, UK

${ }^{\mathrm{b}}$ Department of Chemical Engineering, McGill University, Montreal, QC, H3A 0G4, Canada

c Department of Chemical Engineering, University of Waterloo, 200 University Ave. W, Waterloo, ON N2L 3G1, Canada

Keywords: Relative Diffusivity, In-plane, Thin Porous Media, Fuel Cell, Gas Diffusion Layer

${ }^{*}$ Corresponding Author:

Professor William Gale

Centre for Integrated Energy Research,

School of Chemical \& Process Engineering,

University of Leeds, Leeds, LS2 9JT, UK

Email:W.F.Gale@leeds.ac.uk 


\section{Abstract}

A new experimental technique, extended from similar work on dry materials, is presented for measuring the in-plane components of the relative diffusivity tensor for partially saturated porous media. The method utilizes a custom-built holder and measures the transient response to oxygen concentration changes at the boundaries of a porous sample placed between two plates surrounded by a cooling block. The apparatus is kept close to the freezing temperature of water to ensure stable saturation throughout the experiment. Fick's second law is used to fit the transient change in concentration to a numerical solution to obtain the diffusion coefficient for samples of differing saturation. As expected the effective gas diffusivity is found to decrease with increasing water saturation of the media as the porosity is reduced and the tortuosity of the diffusion pathways increased. After extensive validation, this new technique is used to determine the relative in-plane diffusivity of some common fuel cell gas diffusion layer materials. The results are found to follow a power-law function dependent on the saturation consistent with previous modelling work. 


\section{Introduction}

Transport in thin, partially saturated porous media is of interest to fuel cell engineers due to the use of thin porous materials known as gas diffusion layers (GDL) in polymer electrolyte fuel cells (PEFC). Water produced by the reaction on the cathode side of the PEFC can form liquid which percolates throughout the GDL, blocking reactant gases and reducing performance through mass transport limitations [1]. The GDL has been intensively studied to date, but mostly with respect to single-phase transport properties [2-9]. To reduce stack costs, cell manufacturers aim to increase power density, which in turn increases water production within the cell. Under these conditions, the GDL can become partially filled with liquid water owing to the condensation of water vapour if the relative humidity exceeds the saturation point. If ameliorating mechanisms are not in place, such as purging or heating, the reactant diffusion through the media becomes significantly hindered compared to dry conditions. An understanding of the relative diffusivity, i.e. how gases diffuse through the partially water-filled porous media, is essential for understanding and improving the performance of the fuel cell.

The typical material used as GDLs is a carbon fibre paper which exhibits anisotropic transport characteristics. Fibres have a high degree of in-plane (IP) alignment, increasing transport in this direction compared with through-plane (TP). The TP direction tends to be the focus of studies, as this is the principal direction for transport from the gas channels to the reaction sites. However, IP transport is also important for distributing gases beneath the flow-field ribs, facilitating a more uniform currentdensity across the cell, and increased durability [7,10]. Channel design and 
implications are reviewed by Hamilton \& Pollet [11]. To make the GDLs more hydrophobic and therefore improve water management they are often treated with PTFE. Flückiger et al. showed that PTFE treatments reduce diffusive transport by occupying and blocking the pore pathways [4], but this reduction is a worthwhile tradeoff since it prevents liquid water from spreading within the GDL where it would completely block gas diffusion.

A review of the experimental and modelling techniques used to characterize the diffusive transport in PEFC materials is presented by Ismail et al. [12]. Numerous techniques have been employed to measure the dry diffusivity in both IP and TP directions. Büchi and co-workers used electrochemical impedance spectroscopy (EIS) to measure the ionic transport in an electrolyte soaked GDL, and by analogy diffusive transport [5], [13], [14]. This technique is not readily adaptable to partially saturated porous media, due to problems establishing a two-phase liquid-liquid solution. However, numerous in-situ techniques can be applied to diagnose mass transfer in running fuel cells, such as EIS and limiting current measurements [15] employing measurements coupled with models to extract the contributions of each component to mass transfer limitations. However, with in-situ techniques, knowledge and/or control of the water saturation becomes more difficult, and setups must also be combined with expensive visualisation techniques such as $\mathrm{x}$-ray radiography [16]. Another method is the Loschmidt cell, which measures the transient concentrations of a mixture within two chambers separated by a porous sample and/or controllable barrier [17], [18]. This method has not been applied in the IP direction, although the approach presented by Rashapov et al. [19] is similar; a step change in concentration boundary conditions is applied and the transient concentration of oxygen is measured. 
To date, only a few studies have succeeded in measuring the diffusivity in GDLs under conditions of variable water saturation [20], [21], [22]. These studies all focused on the TP direction, but to fully understand transport within the GDL it is also necessary to measure the IP component(s) of the diffusivity tensor. These methods are unsuitable for IP measurements, however, since they require the use of a reactive layer on one face of the GDL to consume the transferring species resulting in a TP diffusive flux. Numerous studies have approached the problem by modelling transport using direct numerical simulation on images of the GDL microstructure. Becker et al. obtained tomographic images of dry GDLs, then simulated water invasion using morphological image opening, followed by diffusion calculations in the remaining gas space [23]. The problem with this approach is that simulating water invasion in mixed wettability, fibrous, anisotropic media is not trivial. Garcia-Salaberri et al. performed LatticeBoltzmann simulations on tomographic images with water injected during imaging, hence providing realistic invasion patterns [24]. A key finding of that work and the subsequent work [25] was that global average water saturation was not a good indicator of diffusive resistance. The presence of saturation gradients undermined any attempts to extract generalized trends about relative diffusivity. Garcia-Salaberri et al. also recommend that diffusion measurements are made under conditions of uniform saturation profile.

The aim of the present paper is to demonstrate an experimental methodology, which could be applied to any thin porous media, to evaluate the relative IP diffusive transport in the presence of water-filled pores. The method utilizes an oxygen sensor placed 
inside a custom-built sample holder that enables the control of temperature and oxygen concentrations at the outer boundaries of the samples. Saturation is carefully controlled and regulated by keeping the water in a state close to freezing. The transient response to a step-change in oxygen concentration at the boundaries is used to calculate diffusivity using Fick's second law. The present study is the first to experimentally examine the in-plane diffusive transport through the GDL as a function of water saturation.

\section{Scientific Approach}

\subsection{Theoretical Background}

\subsubsection{Relations for Dry Material}

The general conservation of mass equation for a species in a fluid passing through porous media, neglecting sources since no reactions or phase changes are taking place and assuming incompressible flow, is given as:

$$
\frac{\partial(\phi C)}{\partial t}=-\nabla \cdot\left(C \boldsymbol{u}_{s}-D_{E F F} \nabla C\right)
$$

where $\phi$ is the porosity of the medium, $\boldsymbol{u}_{\boldsymbol{s}}$ is the superficial velocity $\left(\boldsymbol{u}_{\boldsymbol{s}}=\phi \boldsymbol{u}\right), C$ is the concentration or volumetric fraction in the fluid phase $\left(\mathrm{mol} \mathrm{m}^{-3}\right)$, and $\boldsymbol{D}_{\boldsymbol{E F F}}$ is a general effective diffusion-dispersion tensor defined as:

$$
\boldsymbol{D}_{\boldsymbol{E F F}}=\phi D_{A B} \boldsymbol{I}
$$


where $I$ is the identity tensor, $D_{A B}$ is the molecular diffusion coefficient of species $A$ through stagnant species $B$ and is assumed to be spatially constant, as the sample holder temperature is held constant. The GDL is assumed to be isotropic in the crosssectional plane normal to diffusion. We also assume a zero pressure gradient across the sample and attribute all motion to diffusion so that Equation (2) becomes a scalar: $D_{E F F}=\phi D_{A B}$. Combining Equation (2) into Equation (1), and also simplifying to its one dimensional form, known as Fick's $2^{\text {nd }}$ Law of Diffusion, we have:

$$
\frac{\partial(C)}{\partial t}=D_{A B} \nabla^{2} C
$$

Note that $\phi$ has now disappeared because the impact of the reduction in pore volume on the time-dependent accumulation term (LHS) and the reduction in flux of species in $D_{E F F}$ (RHS) cancel each other. Shen and Chen [26] warn that cancelling of the porosity term leads to identical concentrations at a given point in space and time regardless of the material's porosity, an incorrect result. This implies that the effective diffusion coefficient must also scale inversely with tortuosity like:

$$
D_{E F F}=D_{A B} \frac{\phi}{\tau_{\phi}}
$$

where tortuosity, $\tau_{\phi}$, represents the increase in diffusion path length in the presence of obstacles in the form of the solid phase and is defined here as:

$$
\tau_{\phi}=\left(\frac{\Delta l}{\Delta x}\right)^{2}
$$


where $\Delta l$ is the diffusion path length and $\Delta x$ is the physical length of the medium. Using the effective diffusion coefficient, Equation (3) becomes:

$$
\frac{\partial(C)}{\partial t}=\frac{D_{A B}}{\tau_{\phi}} \nabla^{2} C=D^{\prime} \nabla^{2} C
$$

Here the notation $D^{\prime}$ is used to represent a reduced diffusion coefficient, dependent only on tortuosity. There is much confusion surrounding the definition of tortuosity, owing to the inconsistent mathematical treatments throughout the literature, as documented by Epstein [27]. It is common in fuel cell literature to express the normalised effective diffusivity of dry materials purely in terms of porosity, such as:

$$
\frac{D_{E F F}}{D_{A B}}=f(\phi)=\frac{\phi}{\tau_{\phi}}
$$

since the tortuosity is some function of porosity. A common expression for $\tau_{\phi}$ is $\phi^{-0.5}$ leading to: $f(\phi)=\phi^{1.5}$. Other common expressions for $f(\phi)$ are presented by Shen and Chen [26] and Hwang and Weber [20].

\subsubsection{Extension to Partially Saturated Material}

When considering a partially saturated domain, liquid can be treated in a similar fashion to the solid phase, with the effect of both reducing the pore volume and increasing the tortuosity. So if saturation, $S$, is the fraction of the pore volume occupied by water, then all the $\phi$ terms can be multiplied by $(1-S)$ and the effective diffusivity can be formulated as follows: 


$$
\frac{D_{E F F}}{D_{A B}}=f(\phi) \cdot g(S)=\frac{\phi}{\tau_{\phi}} \cdot \frac{(1-S)}{\tau_{S}}
$$

In Equation (8) we have decoupled the contributions of gas phase blockages, from solid and liquid respectively, into: 1) volume reduction as the numerators and 2) tortuosity increase as the denominators. When Equation (8) is substituted into Equation (1) the following is obtained:

$$
\frac{\partial(C)}{\partial t}=\frac{D_{A B}}{\tau_{\phi} \tau_{S}} \nabla^{2} C=D_{S}^{\prime} \nabla^{2} C
$$

Note that as before, where porosity related terms cancel for Equation (6), so do the saturation's contributions to a reduction in pore volume. The transient experiment only measures the reduced diffusion coefficient $\left(D_{S}{ }^{\prime}\right)$ due to an increase in tortuosity i.e. diffusional path-length. Therefore, by normalising the measured reduced diffusion coefficients, we obtain the saturation dependent tortuosity:

$$
\tau_{S}=D^{\prime} / D_{S}^{\prime}
$$

This relation is often described using a power-law function such as:

$$
\tau_{S}=(1-S)^{-m}
$$

Therefore, the combined saturation dependent function in Equation (8) can be expressed as follows, with $n=1+m$ : 


$$
g(S)=\frac{(1-S)}{\tau_{S}}=(1-S)^{n}
$$

This is the familiar form used throughout the fuel cell literature, but it must be remembered that it includes both saturation dependent modifications to the effective diffusion coefficient. This is particularly important when used in transient simulations, where the reduced coefficient should be used.

\subsection{Experimental Setup}

The method for measuring the diffusivity of the porous material is based on that of Rashapov et al. [19], [28]. The transient response to a step change in oxygen concentration at the boundaries of the sample is monitored at a fixed point in the sample with an optical oxygen sensor (OceanOptics FOXY-Neofox ${ }^{\circledR}$ ).

The previous method set the initial oxygen concentration throughout the sample to zero by purging it with nitrogen and then removing the gas supply, thereby manually returning the boundary conditions to atmospheric concentration of $20.9 \%$. This method was avoided in the present work because the pressure from the purging would possibly disturb the liquid configuration and cause evaporation, thus adding uncertainty to the calculated saturation values. Instead slow purging was used by flowing gases past the ends of the sample at a low flow rate $(50 \mathrm{ccm})$ and waiting for the sample interior to equilibrate with the end conditions via diffusion. A sample holder with small chambers next to the sample edges was constructed for this purpose, and the full experimental set-up is shown in Figure (1). 

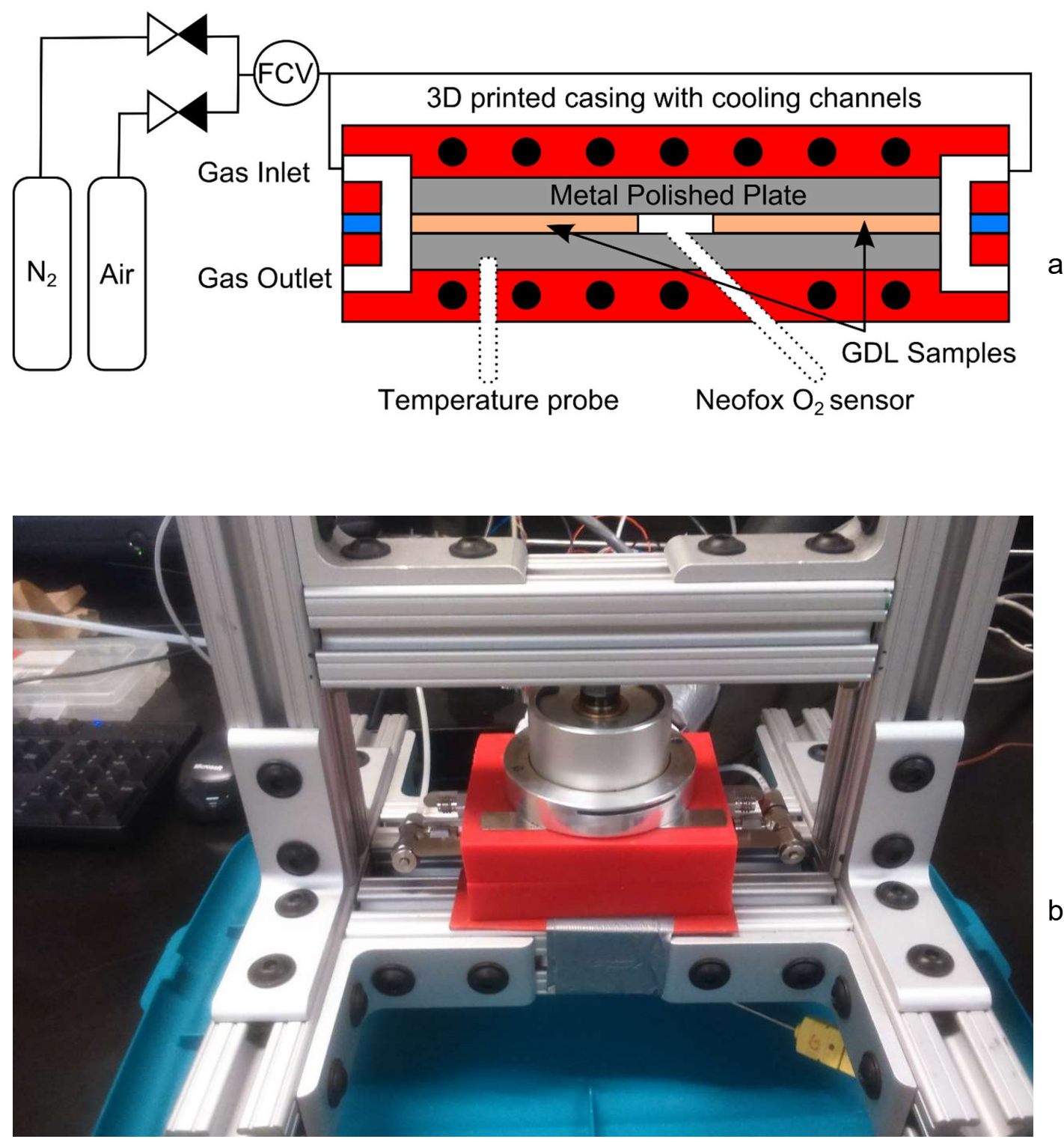

Figure (1): Schematic diagram of the experimental set-up.

Boundary conditions were controlled by combining nitrogen and air with mass flow controllers and passing the flow past the end(s) of the sample. The holder was partially constructed with a 3D printer (B9Creator) to enable the design to include a serpentine cooling channel. This was required for more precise temperature control to ensure that samples retained their saturation throughout the experiment. The cooling channel 
design is shown in Figure (2). The temperature was regulated by constantly pumping cooling fluid with a Fisher Scientific Isotemp 3006D refrigerated circulator set to $-10^{\circ} \mathrm{C}$. With the apparatus exposed to the laboratory atmosphere at room temperature, it was necessary to measure the temperature of the holder as close to the sample as possible. A hole was drilled into the under-side of the sample holder to a depth $1 \mathrm{~mm}$ from the inner surface and a mineral insulated thermocouple was inserted and fastened with epoxy resin. The temperature recorded was between $-1^{\circ} \mathrm{C}$ and $+2^{\circ} \mathrm{C}$ at all times. The longest experiments lasting over an hour, with several changes of oxygen concentration, were found to have transient responses that remained constant, proving that sample saturation was maintained. The state of water could not be verified visually, however, the temperature range measured for the holder meant that water was either in a frozen state, or very cold with a small vapour pressure.

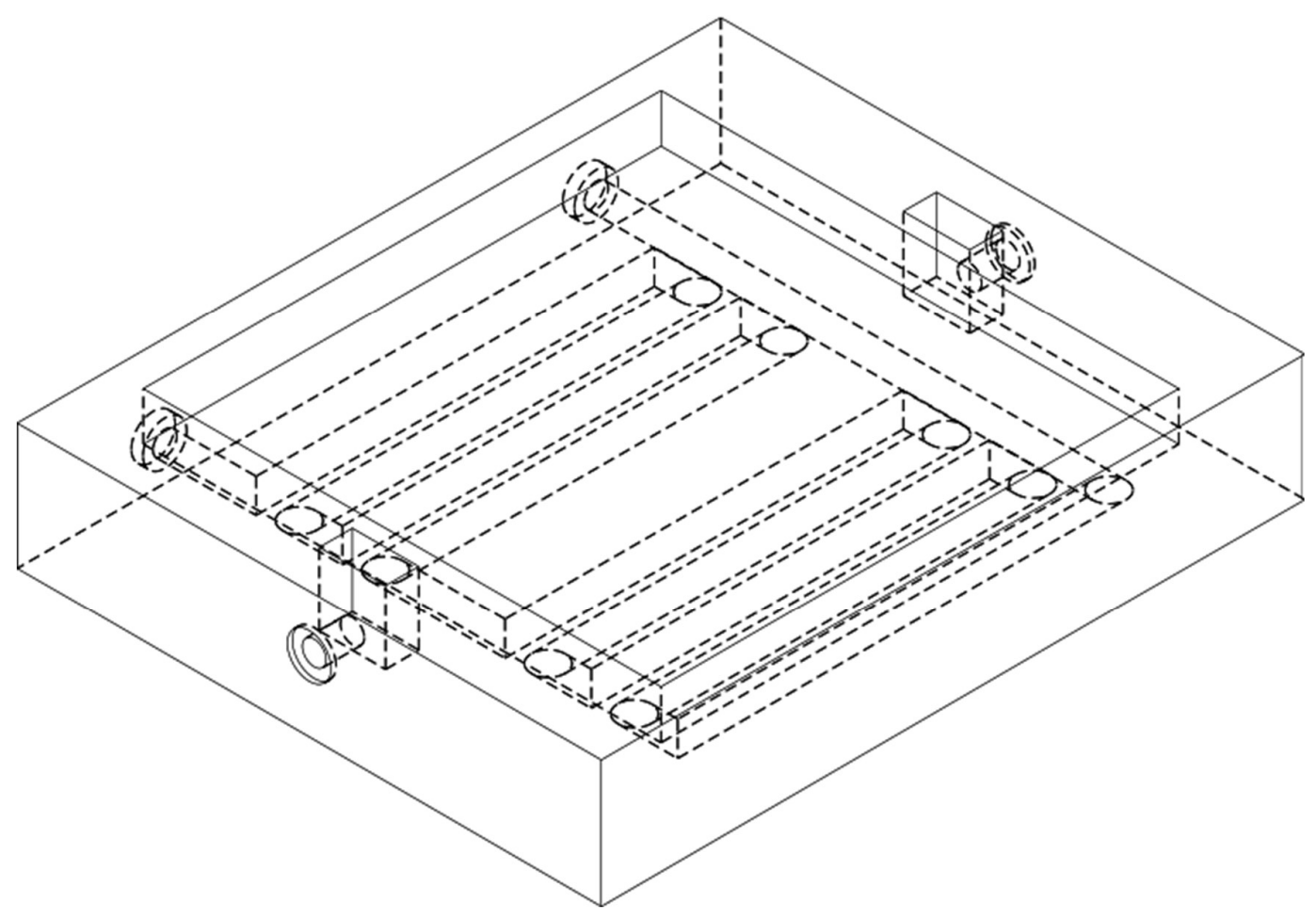

Figure (2): Detailed view of the 3D printed holder section with cooling channels 
The samples were sealed between two steel plates with the printed cooling plates sandwiched on each side. The steel plates were polished to a mirror finish to eliminate surface roughness and any bowing of the surfaces of the plates. This ensured that even contact was made with the sample surfaces. Rough or uneven surfaces would have allowed additional diffusional pathways and introduced systematic errors. Pliable putty was used to encircle the samples and the boundary gas chambers as a sealant and this was rolled by hand to a thickness of about $1 \mathrm{~mm}$. Care was taken to ensure that the putty was rolled evenly and was placed as close to the samples as possible to prevent air gaps along the sample length.

\subsection{Sample Preparation}

Samples were cut to a length of either 30 or $70 \mathrm{~mm}$, for the single or double-ended setup, and weighed while dry. They were then submerged in a beaker of de-ionized water (18 $\mathrm{M} \Omega$ ) and vacuum pumped for several minutes to remove any trapped gas. The samples were then dried, with all faces exposed to the atmosphere, on a mass balance to a predetermined target weight to achieve the desired saturation $(S)$, defined by the following expression:

$$
S=\frac{M_{\text {wet }}-M_{d r y}}{\phi V \rho_{\text {water }}}
$$

where $M_{w e t}$ and $M_{d r y}$ are the mass of the samples when wet and dry, $\phi$ is the sample porosity which were measured in a previous study [29], $V$ is the volume of the sample

when compressed by the holder and $\rho_{\text {water }}$ is the density of water. On initial weighing 
after submersion, the mass of all samples was found to be that of a fully saturated sample. The process of drying water from a hydrophobic media is equivalent to air imbibition, which is known to be less influenced by surface effects and access limitations due to the formation of thin films by the wetting fluid [30]. Consequently, this water configuration within the GDLs is expected to be more uniform which is preferred over injecting liquid water from the surface, as pointed out by García-Salaberri et al. $[24,25]$. In future, it would be desirable to have greater control over the saturation distribution.

The effect of compression on the flow behaviour of the samples was not investigated in this work, so the spacer shims were chosen with a thickness similar to that of the samples to avoid excessive reduction in pore space when clamping and sealing the experiment.

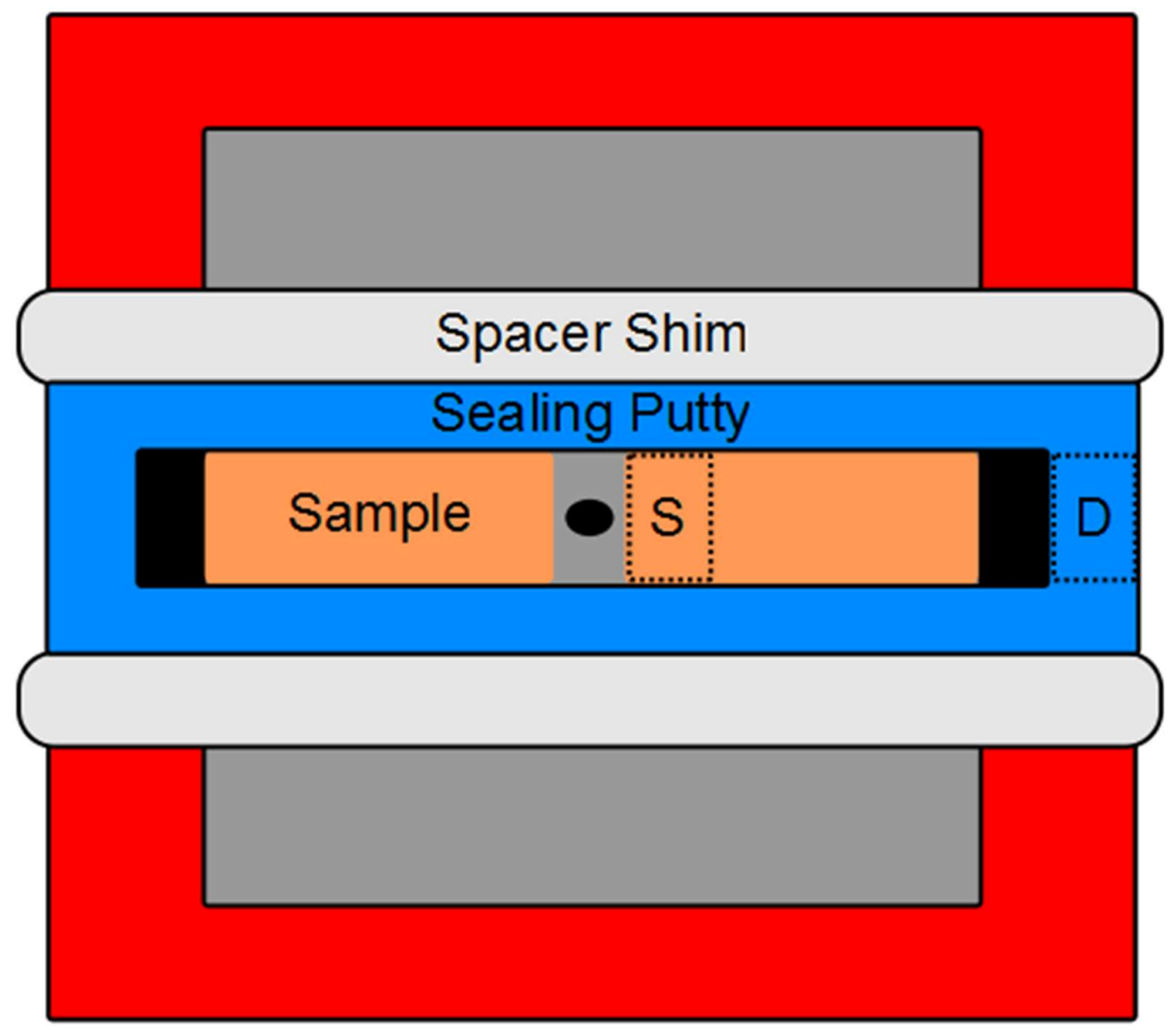


Figure (3): Top-down view of the sample sitting on the lower holder plate. "S" and "D" mark the position of the sealing putty for the single and double-ended setup.

A top-down view of the sample sitting on one of the holder plates is shown in Figure (3). The shims were placed about $10 \mathrm{~mm}$ from the samples in order to let the putty expand away and not intrude into the sample. Samples were not placed directly on top of the sensor to prevent the formation of ice on the tip which would have impeded the measurements. This air gap in the system caused complications to the analysis which are discussed further below. The holder was kept at zero degrees when transferring the sample and changing time is less than 1 minute with the bare surface of the holder exposed for about 10 seconds. Some condensation from the atmosphere is possible in this time and care was taken to minimize this with surfaces wiped at the latest point possible. Evaporation from the sample is minimized by previously freezing it and transferring from freezer to holder in minimal time. Results repeated over the course of an hour gave very similar diffusivities indicating that saturation is constant throughout the experiment.

The experiments were conducted in one of two equivalent (i.e. symmetrical) arrangements. These are single and double-ended, with the position of the putty marked with an 'S' and ' $D$ ' in Figure (3), respectively. For the double-ended setup, gas was flowed past both ends of the sample and the sample was cut in half and placed on each side of the sensor. Alternatively, gas was only flowed past one end of the sample while the other was blocked, in which case the sample was only placed on one side of the sensor. In both cases the sensor was located at the no flux position in 
the domain, and the mathematical treatment was identical, with minor differences in the domain length and relative position of the sensor. The advantage of the singleended setup is to ensure that pressure differentials do not exist across the sample, so that all transport can be attributed to diffusion. However, results repeated for both setups yielded similar results, giving confidence that the results are not influenced by convective flow. In future, the double ended-setup could be used to simultaneously measure diffusivity and permeability with the appropriate sensors.

\subsection{Calculation of the Effective Diffusion Coefficient}

The following analytical solution to Fick's Second Law, given by Crank [31], was used previously to find the effective diffusion coefficient, $D_{E F F}$ [19] of the GDL by fitting the following equation to the experimental data using $D_{E F F}$ as the only fitting parameter:

$$
\begin{aligned}
\frac{C(t)-C_{0}}{C_{1}-C_{0}}= & \sum_{n=0}^{i n f}(-1)^{n} \operatorname{erfc}\left[\frac{(2 n+1) l-x}{2 \sqrt{D_{E F F} t}}\right] \\
& +(-1)^{n} \operatorname{erfc}\left[\frac{(2 n+1) l+x}{2 \sqrt{D_{E F F} t}}\right]
\end{aligned}
$$

Equation (14) is valid for homogeneous samples which was the case with the previous experiment [19] as the GDL filled the entire sample space. However, this approach was not valid for the present study due to the air gap around the sensor, which created a composite domain. Instead the experiment was modelled as a transient onedimensional diffusion process through each section using a finite volume based PDE solver (FiPy [32]). Equation (6) was solved with the following initial and boundary conditions: 


\begin{tabular}{|l|l|l|}
\hline & Single Ended & Double Ended \\
\hline Initial Condition & \multicolumn{2}{|c|}{$\left.C(x)\right|_{t=0}=C_{0}$} \\
\hline Left B.C & \multicolumn{2}{|c|}{$\left.C(t)\right|_{x=0}=C_{1}$} \\
\hline Right B.C & $\left.\frac{\partial C(t)}{\partial x}\right|_{x=l}=0$ & $\left.C(t)\right|_{x=2 l}=C_{1}$ \\
\hline
\end{tabular}

A uniform one-dimensional mesh with an element length of 1E-04 m was used for the modelling domain and a time-step of 0.25 seconds was found to be sufficient for solution independence of numerical factors. In the air gap around the sensor the bulk diffusivity of oxygen in air was used as the diffusion coefficient. The surrounding section(s) containing sample were assigned an initial guess for the diffusivity of the GDL by first fitting the experimental data using Crank's Equation (14). The SciPy optimization package [33] was then used to find a closer approximation of the effective diffusivity for the sample by minimizing the following objective function using the 'LBFGS-B' method (scipy.optimize.fmin___bfgs_b):

$$
O b j=\sum_{t=0}^{t_{\max }}\left(\frac{C(x)_{\text {exp }}-C(x)_{\text {num }}}{C(x)_{\text {num }}}\right)^{2}
$$

Which is a sum of the square of the percentage difference in concentration at the sensor point between the experimental and transient response simulated by the PDE solver, for all times during one experiment. Bounds for the diffusivity of the GDL were assigned using half and double the initial guess. The sensor captures data every second and the longest experiment typically lasts for around 1000 seconds. The 
transient diffusion equation was solved implicitly with a time-step of 0.25 seconds, so the longest simulations must solve the transient diffusion equation up to 4000 times per iteration. The convergence criteria used for the optimization was met when the maximum component of the projected gradient reduced to below a tolerance of $5 \mathrm{E}$ 06, which was found to reduce the objective function sufficiently to give excellent agreement between simulation and experiment. A plot of the results for a single fitting process is shown in Figure (4) and the convergence data is shown in Figure (5), showing a minimum is reached. Typically no more than 15 iterations were required to converge, meaning the process completes in a matter of minutes per data-set.

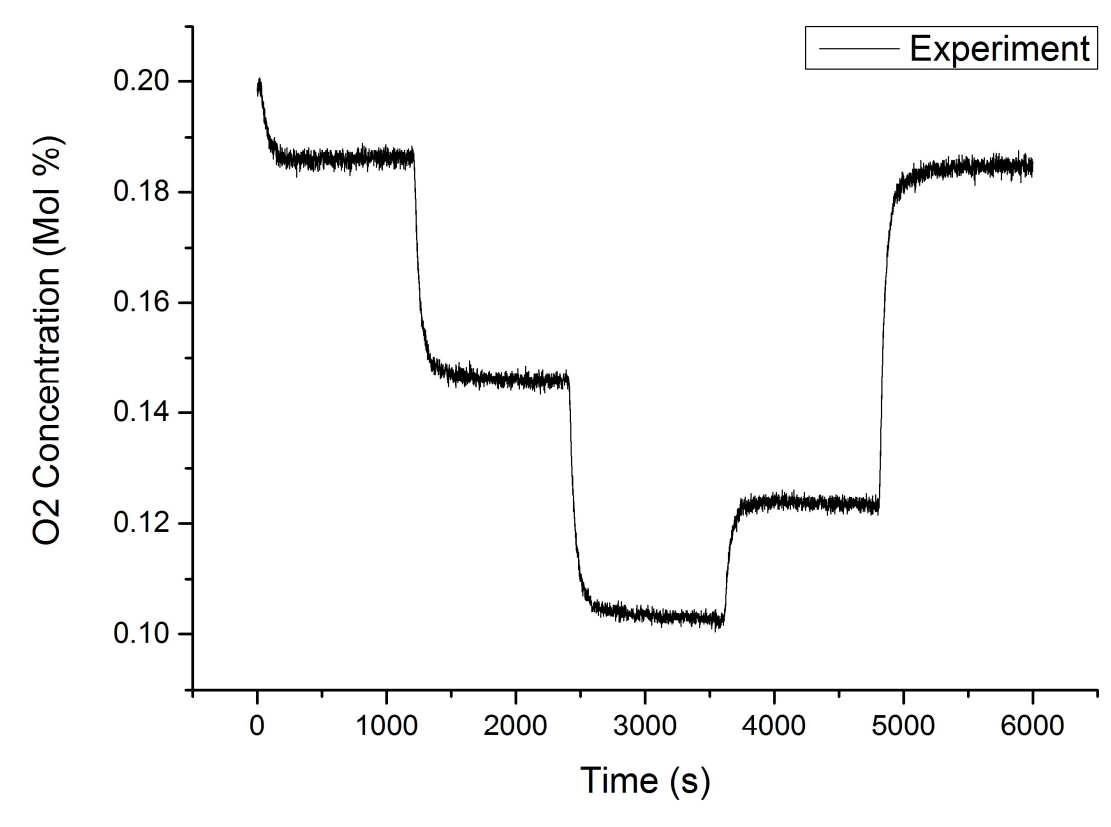




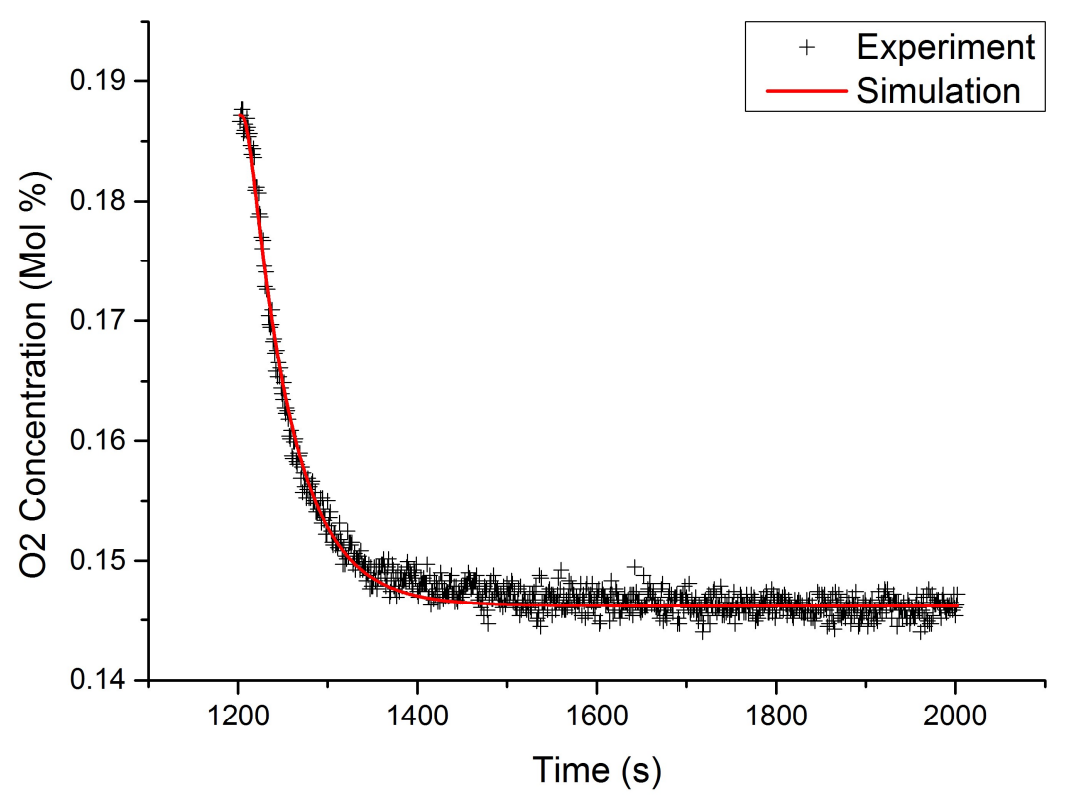

Figure (4): Time-series plot for dry sample of SGL 10BA with 5\% PTFE showing (a) the cycling of changes in flowing gas concentration and (b) a section of the time-series with fitted simulation data. 


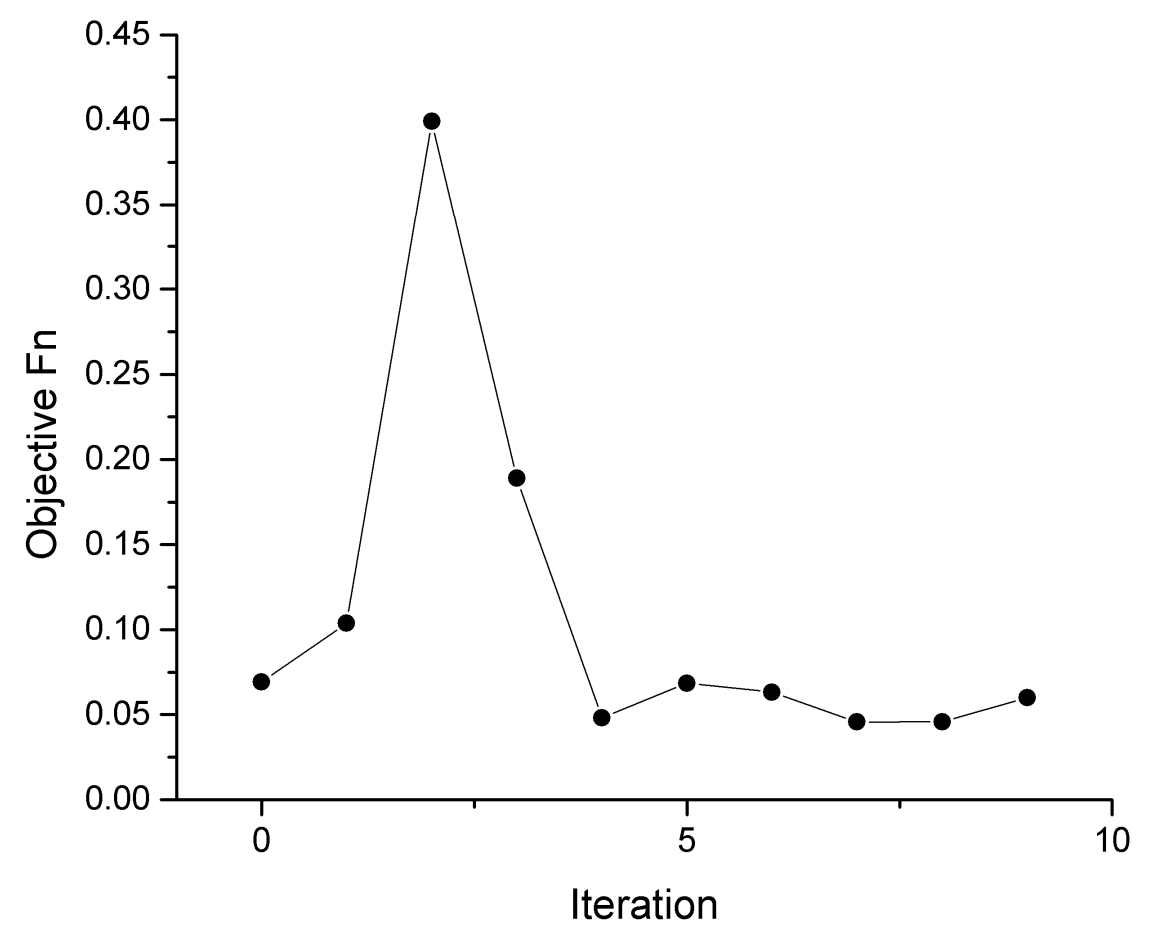

a

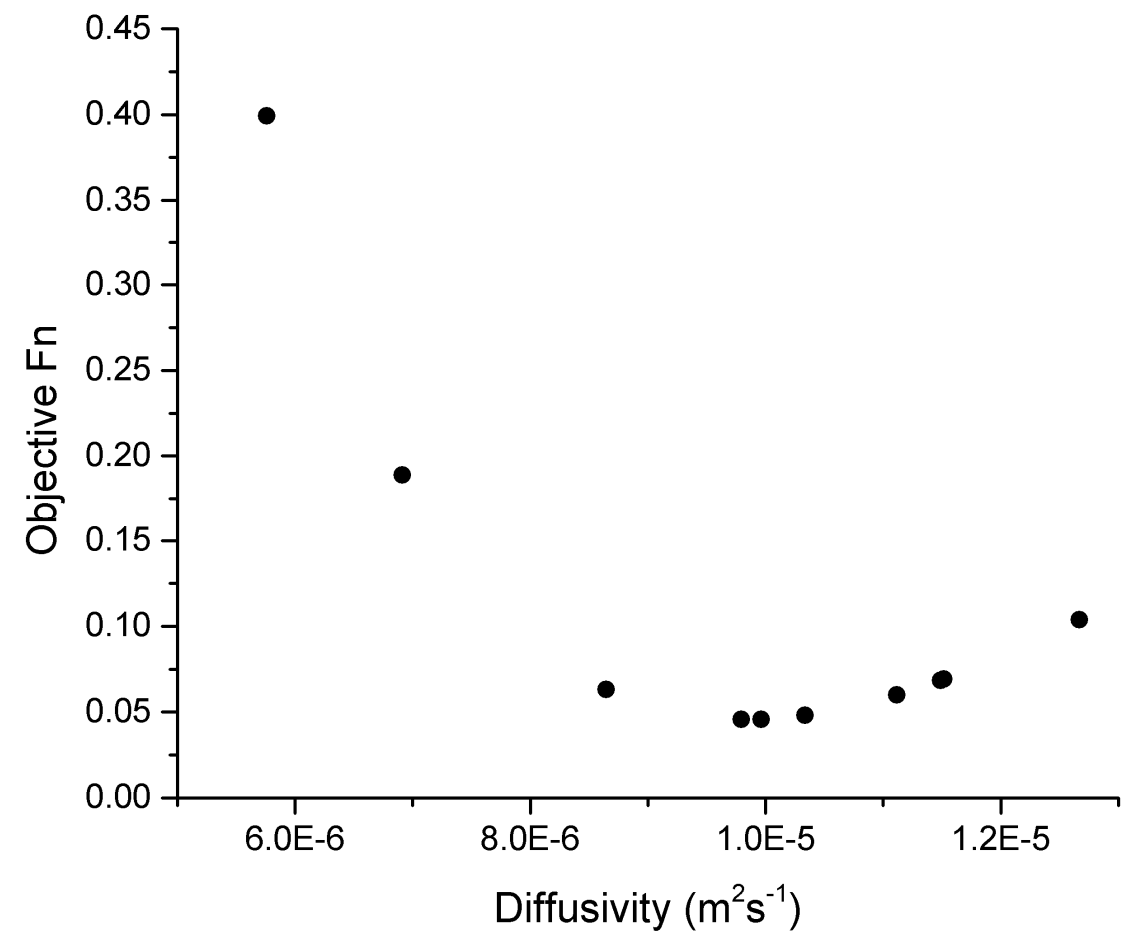

b 
Figure (5): Convergence data for SGL 10BA with 5\% PTFE showing (a) the objective function for each iteration of the minimization (b) the objective function vs. diffusivity showing a minimum in the parameter space is found.

Other studies monitoring transient diffusion through multiple domains each containing different materials or inhomogeneous spaces have employed an equivalent resistance assumption. Diffusivity of the individual domains is then extracted through a resistors in series calculation [34]. This assumption can introduce significant error to the calculation of the component diffusion coefficients for transient cases. The error is greater when the diffusivity of the domains differs substantially, or the lengths of the different sections are comparable, so that no material dominates the process. If it is assumed that diffusion takes place through a homogenous region with a single averaged diffusion coefficient, a smooth concentration profile is produced, whereas a composite domain has a different profile in each section and this affects the response over time. As the sensor only measures concentration at a fixed position, an accurate prediction of the concentration profile and its time dependence at each position is therefore important. Transient diffusion in such situations is a tricky subject, summarized by Crank [31], where elaborate equations describing the time lag for concentration changes have been provided for special circumstances. Other forms of analytical solution are available such as those using eigenfunctions [35]. However, given the computational efficiency of the current numerical procedure, it seems to be adequate. The script used to fit the data is provided as supplementary material with both methods available for comparison. Fitting the experimental data with the analytical solution provided by Crank based on an equivalent resistance underpredicts the diffusion coefficients by as much as $25 \%$ for highly saturated samples. 


\section{Method Validation}

\subsection{Comparison to Previous Studies with Dry Samples}

To ensure that the methodology is valid the results obtained for a dry sample of Toray 090 are shown in detail and compared with previous studies [28,36]. Figure (4) shows the detailed time-series plot generated by the oxygen sensor after being converted to an oxygen concentration. The sensor actually records a phase shift in a pulsed laser which is dependent on the partial pressure of oxygen and also the temperature. Therefore calibration of the sensor is required using known concentrations of gas and polynomial fitting is used to convert the phase shift to an actual concentration reading during the experiment. Mass flow controllers were used to alter the concentration at the sample boundaries and these were calibrated using the bubble tube technique. A second check was also made by exposing the sensor to gas with $7 \%$ oxygen concentration supplied by bottle, but bottles of all the various concentrations used in calibration were not available.

Five different GDL materials were investigated to assess whether structural differences and PTFE coating made a significant difference to the relative diffusivity. The characteristics of the samples, as measured by Rashapov et al. [29] is shown in Table (1).

\begin{tabular}{|c|c|c|c|c|c|c|c|}
\hline Material & PTFE & Porosity & Thickness & Shim & Compres & Dry & Literature \\
& $(w t-$ & $\left(\phi_{o}\right)[29]$ & $\left(\delta_{0}\right)[\mu \mathrm{m}]$ & Thick & sed & Normalized & Value(s) \\
& $\%)$ & & {$[29]$} & ness & Porosity & Diffusivity & \\
& & & & & & \\
\hline SGL 10 & 5.0 & $0.871 \pm$ & $423 \pm 14$ & 406 & $0.866 \pm$ & $0.47 \pm 0.03$ & $0.463[20]$ \\
BA & & 0.006 & & & 0.039 & & \\
\hline
\end{tabular}




\begin{tabular}{|c|c|c|c|c|c|c|c|}
\hline SGL 34 & 5.0 & $0.827 \pm$ & $284 \pm 12$ & 254 & $0.807 \pm$ & $0.54 \pm 0.04$ & $0.53[28]$ \\
BA & & 0.008 & & & 0.036 & & \\
\hline Toray & 0.0 & $0.745 \pm$ & $280 \pm 9$ & 254 & $0.719 \pm$ & $0.52 \pm 0.04$ & $0.54[28]$, \\
090 & & 0.008 & & & 0.032 & & $0.31[36]$ \\
\hline Toray & 5.0 & $0.719 \pm$ & $262 \pm 10$ & 254 & $0.710 \pm$ & $0.51 \pm 0.04$ & $0.56[28]$ \\
090 & & 0.010 & & & 0.032 & & \\
\hline Toray & 5.0 & $0.746 \pm$ & $364 \pm 9$ & 305 & $0.697 \pm$ & $0.34 \pm 0.02$ & $0.49[28]$, \\
120 & & 0.007 & & & 0.031 & & $0.325[20]$ \\
& & & & & & & \\
\hline
\end{tabular}

Table (1): Material Properties and dry diffusivity normalized by open air value with comparison to literature.

The effective dry diffusivity of Toray 090 compressed to a shim thickness, $\delta$, of 254 $\mu \mathrm{m}$ as measured by the experiment is $1.41 \mathrm{E}-05 \mathrm{~m}^{2} / \mathrm{s}$. This value was obtained at a temperature of around $273 \mathrm{~K}$ for which the diffusivity of oxygen through stagnant nitrogen is $1.81 \mathrm{E}-05 \mathrm{~m}^{2} / \mathrm{s}$ in open space according to:

$$
D_{T_{2}}=D_{T_{1}}\left(T_{2} / T_{1}\right)^{1.75}
$$

With a reference value of $2.06 \mathrm{E}-05 \mathrm{~m}^{2} / \mathrm{s}$ at room temperature. As mentioned in Section 2.1.1 the experiment only measures the reduced diffusion coefficient $\left(D^{\prime}\right)$ shown in Equation (6). Therefore, to compare the results with other experiments the normalized value is multiplied by the compressed porosity.

$$
D_{E F F} /_{D_{A B}}=D^{\prime}\left[1-\left(\frac{\delta_{0}\left(1-\phi_{o}\right)}{\delta}\right)\right]
$$


Where the subscript 0 denotes the uncompressed value. This results in a normalized diffusivity of 0.52 for untreated Toray 090 as shown in Table (1) which contains the material properties and dry diffusivity data for all the samples. The results for this experimental setup are within about $10 \%$ of those collected by Rashapov et al. $[19,28]$ using a similar setup where the sample ends were exposed to the atmosphere rather than flowing gasses, who report values between 0.5 and 0.6 . However, a study by Mangal et al. [36] reports values for dry diffusivity for Toray 090 which are about $40 \%$ lower, when comparing values using the compressed porosity. The reported sample thickness and uncompressed porosity values differ between studies and so the reported compressed porosity corresponds to different compression ratios which may account for some of the difference, as well as sample variation and possible material degradation. The SGL values reported in the present study agree well with the literature. However, the present Toray 120 value agrees well with the study of Hwang and Weber [20], who recorded through-plane values, but less well with Rashapov et al. [28], who reported in-plane values. This is a somewhat puzzling result, given that the Toray 090 results agreed so well. We can, at this time, only attribute the difference to material variability.

\subsection{Uncertainty Analysis}

As the diffusivity is determined through fitting with a numerical process, a sensitivity study to determine the uncertainty in the numerical parameters was undertaken. As the process is modelled in one-dimension the parameters that affect the outcome are the sample length and position of the sensor relative to the no-flux position. A $1 \mathrm{~mm}$ variation in both these parameters incurs a $6.5 \%$ difference in predicted sample diffusivity. The normalized tortuosity results presented in the next section calculated 
using Equation (10) using the saturated and dry values are therefore subject to about a $9.2 \%$ uncertainty according to Equation (18). In addition the $g(S)$ value includes a multiplication by a factor of (1-S). We estimate that the uncertainty in $S$ is at most $10 \%$, a full example of the calculations can be found in the Appendix, and so combining errors produces an uncertainty in $g(S)$ around $13.5 \%$ according to Equation (19).

$$
\begin{aligned}
& \left(\frac{\Delta \tau_{S}}{\tau_{S}}\right)^{2}=\left(\frac{\Delta D^{\prime}}{D^{\prime}}\right)^{2}+\left(\frac{\Delta D_{S}^{\prime}}{D_{S}^{\prime}}\right)^{2} \\
& \left(\frac{\Delta g(S)}{g(S)}\right)^{2}=\left(\frac{\Delta \tau_{S}}{\tau_{S}}\right)^{2}+\left(\frac{\Delta S}{S}\right)^{2}
\end{aligned}
$$

Other factors could contribute to systematic errors in the measured diffusivity such as the accuracy of the sensor, the supplied gas concentration which is achieved by mixing air and pure nitrogen, the flow rate of the gases and the presence of dispersion effects and back diffusion in the channel. Attempts were made to eliminate these additional sources of error. The supplied gas concentration is controlled with mixing and, as mentioned in the previous section, the mass flow controllers were periodically calibrated using the bubble-rising technique and performed very stably over long operating periods. The length of the gas supply tubes was kept to a minimum to reduce the time-lag between changing flow rates and recording a change at the sensor, and this was typically a few seconds. The starting point for the data fitting was manually set by visual inspection of the time-series after an initial fit is produced. As the initial change in concentration is quite sharp, this process is quite simple to implement. The gas outlets were exposed to the atmosphere during the experiment but tests were undertaken to establish whether back diffusion was possible from the outlet by comparing results with an outlet submerged in water. No difference in measured transient response was detected for open or submerged gas flow outlets and so back 
diffusion was not present in either case. The flow rate of the gas mixture was always 8.3 $\mathrm{E}-10 \mathrm{~m}^{3} / \mathrm{s}(50 \mathrm{ccm})$. Modelling reveals that this flow rate is sufficient for reducing diffusion effects in the channel, which would effectively increase the diffusion pathways and alter the interpreted diffusivity. However, a systematic experimental study of channel diffusion effects was not performed and is left for a future study.

The repeatability of the results for single samples is very good. Once a saturated sample is enclosed in the holder, if the temperature remains fixed, measured transient responses are repeatable with differences of only a few percent in the calculated diffusion coefficient. Due to time constraints, and also the difficulty in reproducing exact matches in saturation with the current technique for sample flooding and drying, results for each saturation were not repeated using multiple samples of the same material. The saturation method and measurement is certainly an area for improvement in future work and would reduce the uncertainty of the results as the uncertainty of the saturation is a major contributing factor.

\section{Results and Discussion}

\subsection{Gas diffusion through partially saturated samples}

The fitted relative diffusivity data are shown in Table (2). Figure (6) shows the normalised fitted diffusion coefficients for the various samples. The fitted diffusivity is normalized by the value in open space for the corresponding temperature, as with the results in section 3.1 , and the dry diffusivity to retrieve the tortuosity due to saturation only, $\tau_{S}$. The saturation function $g(S)$ is then found by inverting $\tau_{S}$ and multiplying by a factor of $(1-S)$, to account for volume reduction, as per Equation (12). The resulting 
saturation functions are themselves fitted to both Equation (12) to obtain $n$ and the following expression, commonly used in the literature:

$$
g(S)=\frac{1}{2}\left(1+\operatorname{erf}\left[\frac{-\ln (S)+a}{b}\right]\right)
$$

The tortuosity dependence on saturation, Equation (11), is also plotted in Figure (6) with $m=n-1$. At high saturations this relation is sometimes close to the observed values and other times not, especially for Toray 090 with 0\% PTFE, which has a lower tortuosity than predicted. Equation (11) implies that, as the sample becomes fully saturated, tortuosity increases to infinity. Therefore, small deviations from the predicted diffusion rates will be amplified into large differences between observed and predicted tortuosity. This result is perhaps not significant for operating fuel cells as they tend to have maximum saturations far below full saturation. However, it does highlight the sensitivity of the tortuosity to low porosity media in general. For other experiments measuring the full diffusion coefficient with contributions from volume reduction and diffusion path length increase, this sensitivity is perhaps masked. This could be considered an advantage of the experimental procedure presented here, if the connectivity of the pore-space is of primary interest, as the transient response depends inversely on the tortuosity only, as shown by Equation (9).

The data presented is the average result for a number of experiments (typically three) on a single sample collected with different boundary condition changes. All samples were cut from the same sheet along the same direction. Due to time constraints, a study of sheet variability and in-plane directional variability was not possible and is left for future work. In each case the mass flow rates of the gases were altered to change the concentration of oxygen flowing past the sample ends and the system was left to 
reach steady state. The purpose of repeated measurements on the same sample was two-fold. Firstly, the sensor does not measure concentration directly but a phase-shift in a fluorescent response of the coating material on the sensor tip to a pulsed excitation. This phase shift is temperature dependent so in-situ calibration was possible using the various steady-state concentration values. Secondly, for some cases, the calculated diffusivity was found to be different when switching from high to low oxygen concentration boundary conditions and vice versa. This was an indication of a leak, with atmospheric oxygen penetrating the sample holder, thus providing additional diffusion pathways to the sensor. These anomalies were due to inadequate positioning of the sealing putty and results with inconsistent diffusivity were discarded.

SGL 10 BA 5\% PTFE

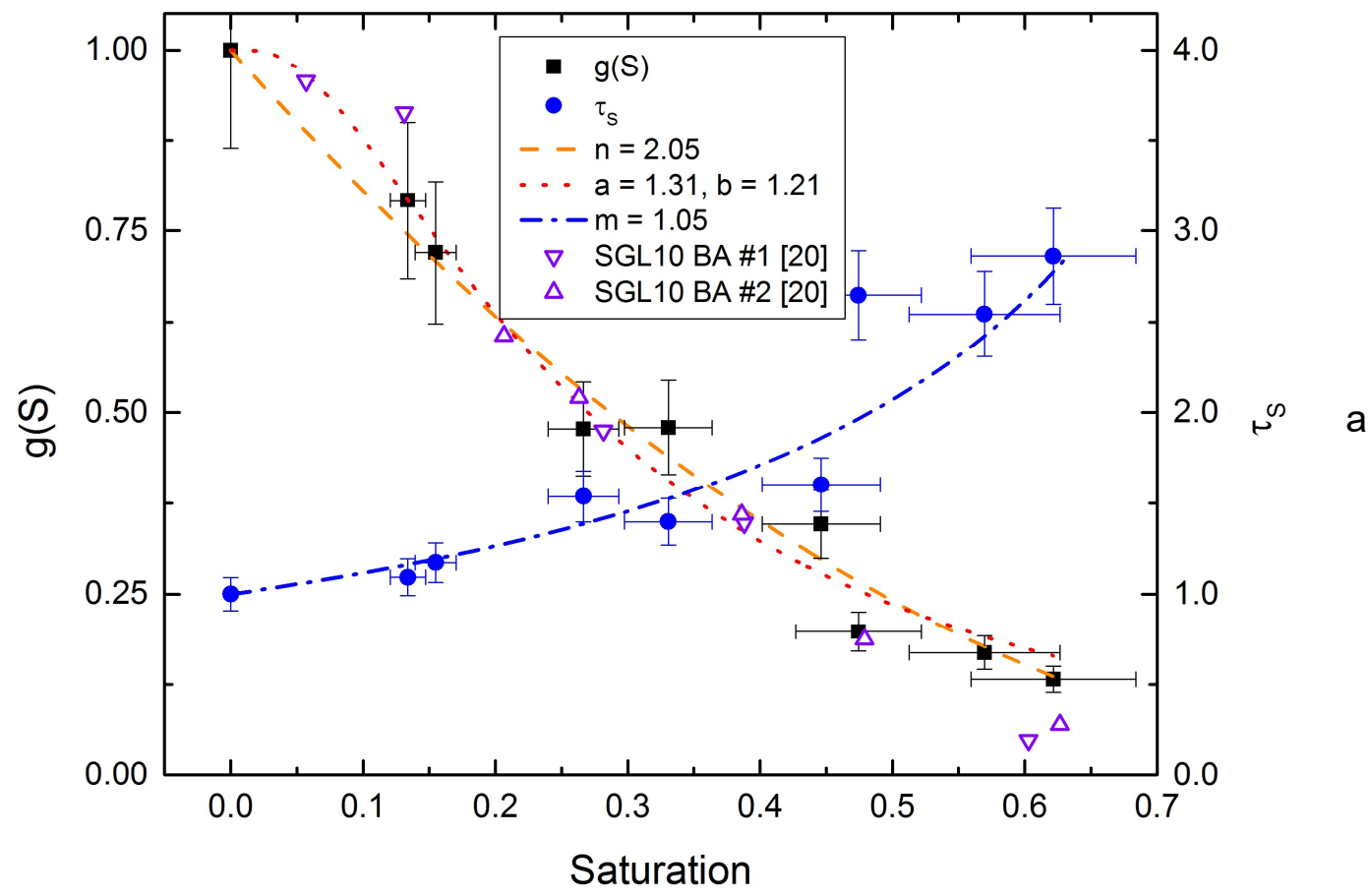


SGL 34 BA 5\% PTFE

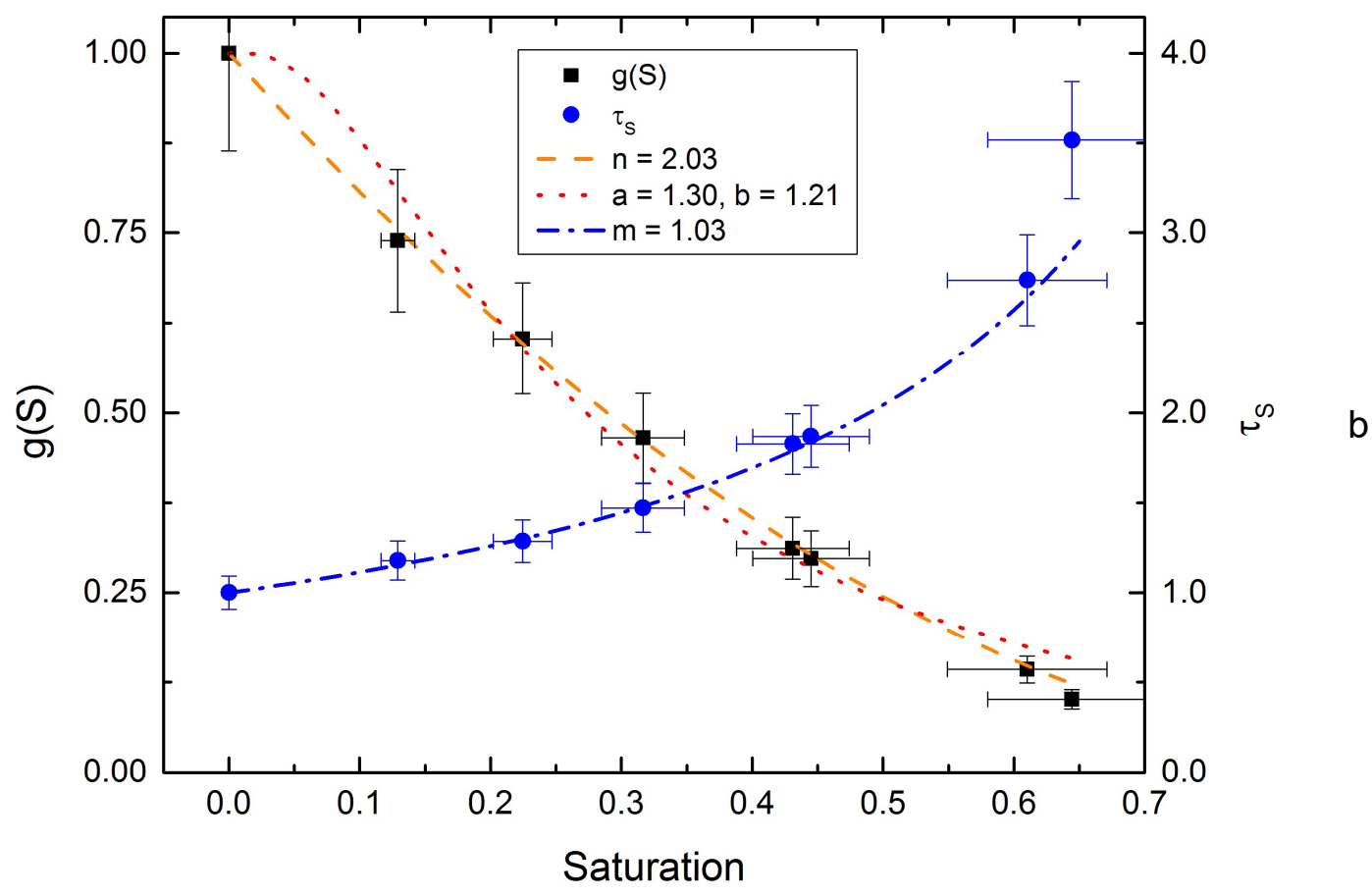

TORAY 090 0\% PTFE

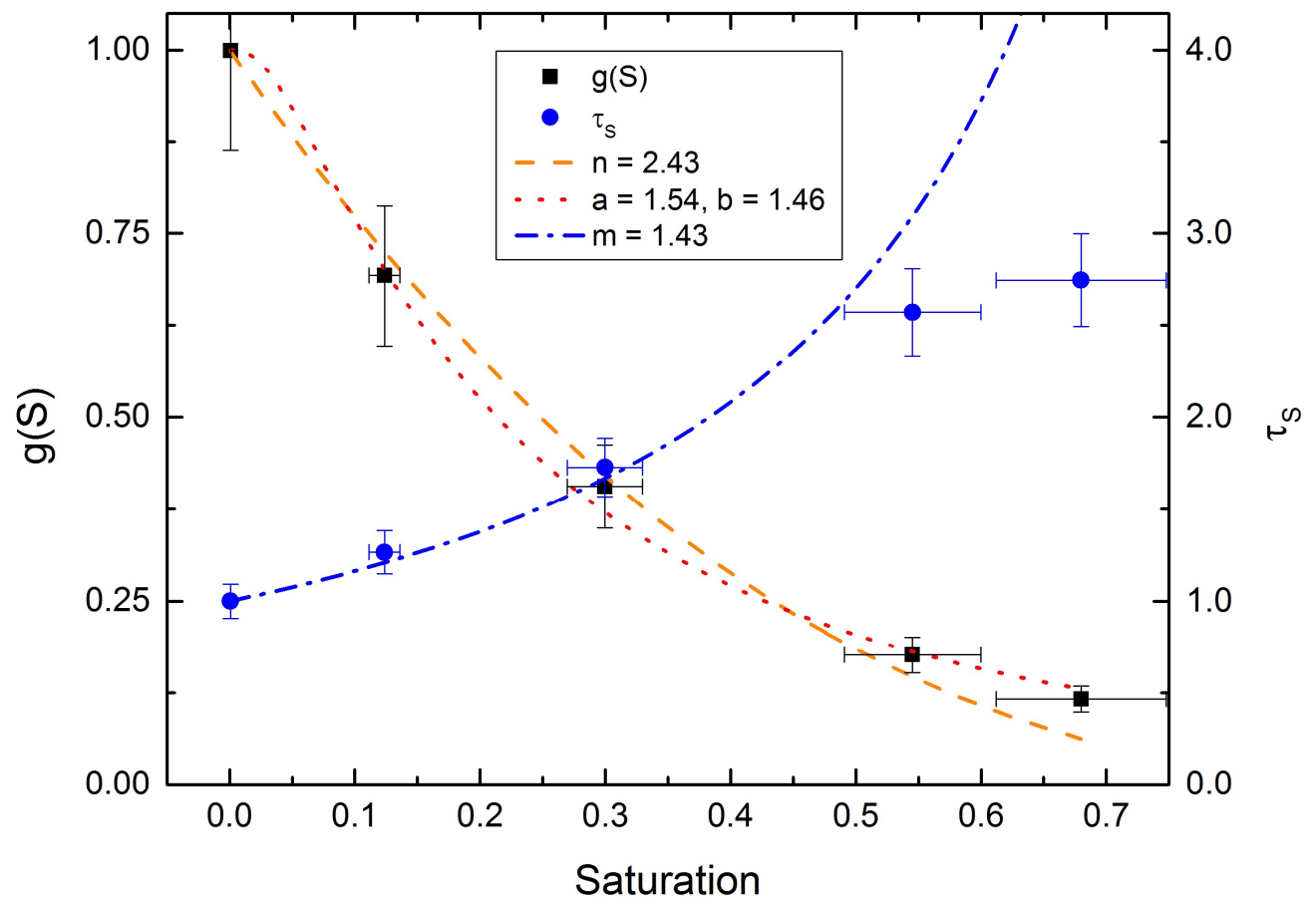

C 


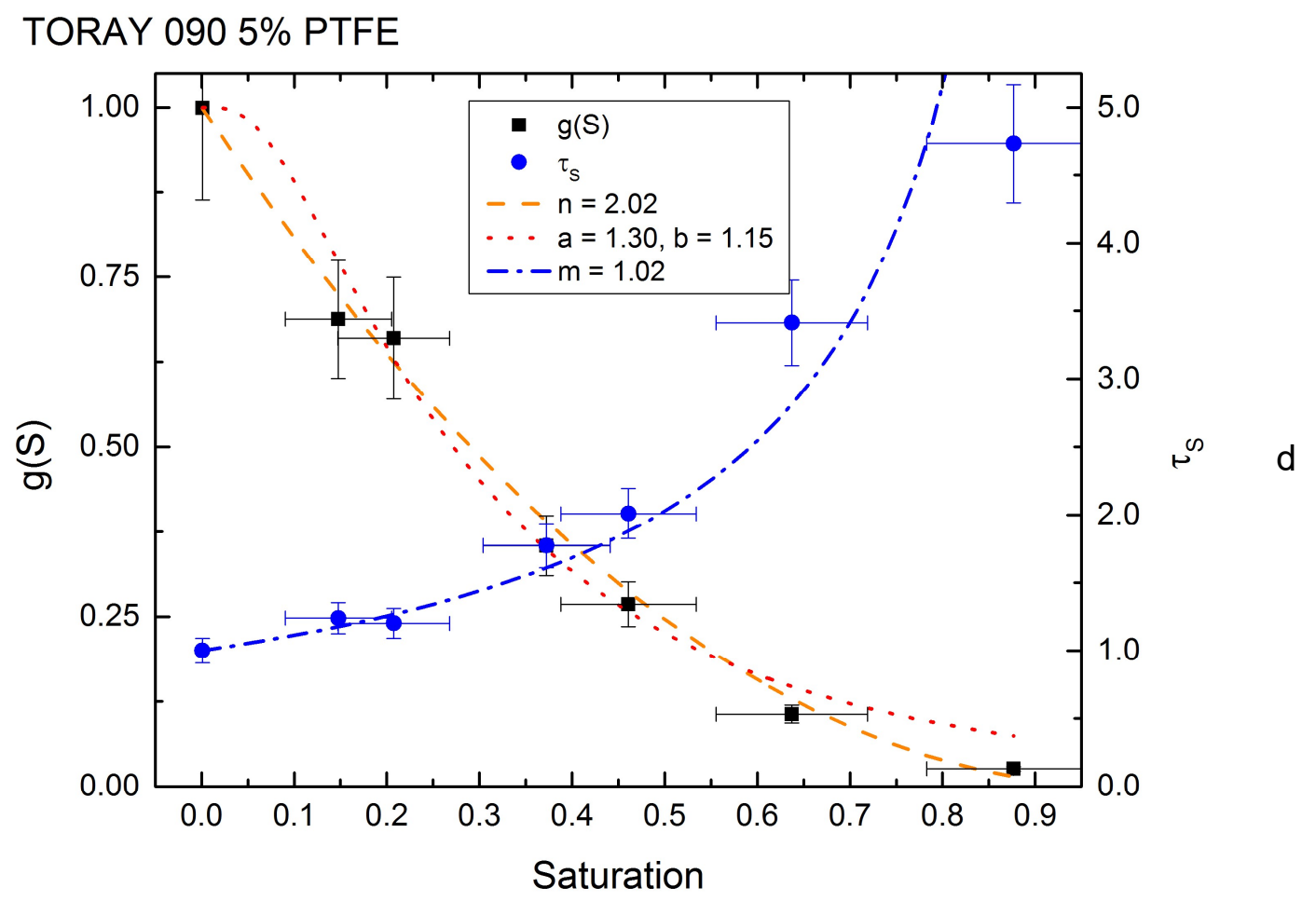

\section{TORAY 120 5\% PTFE}

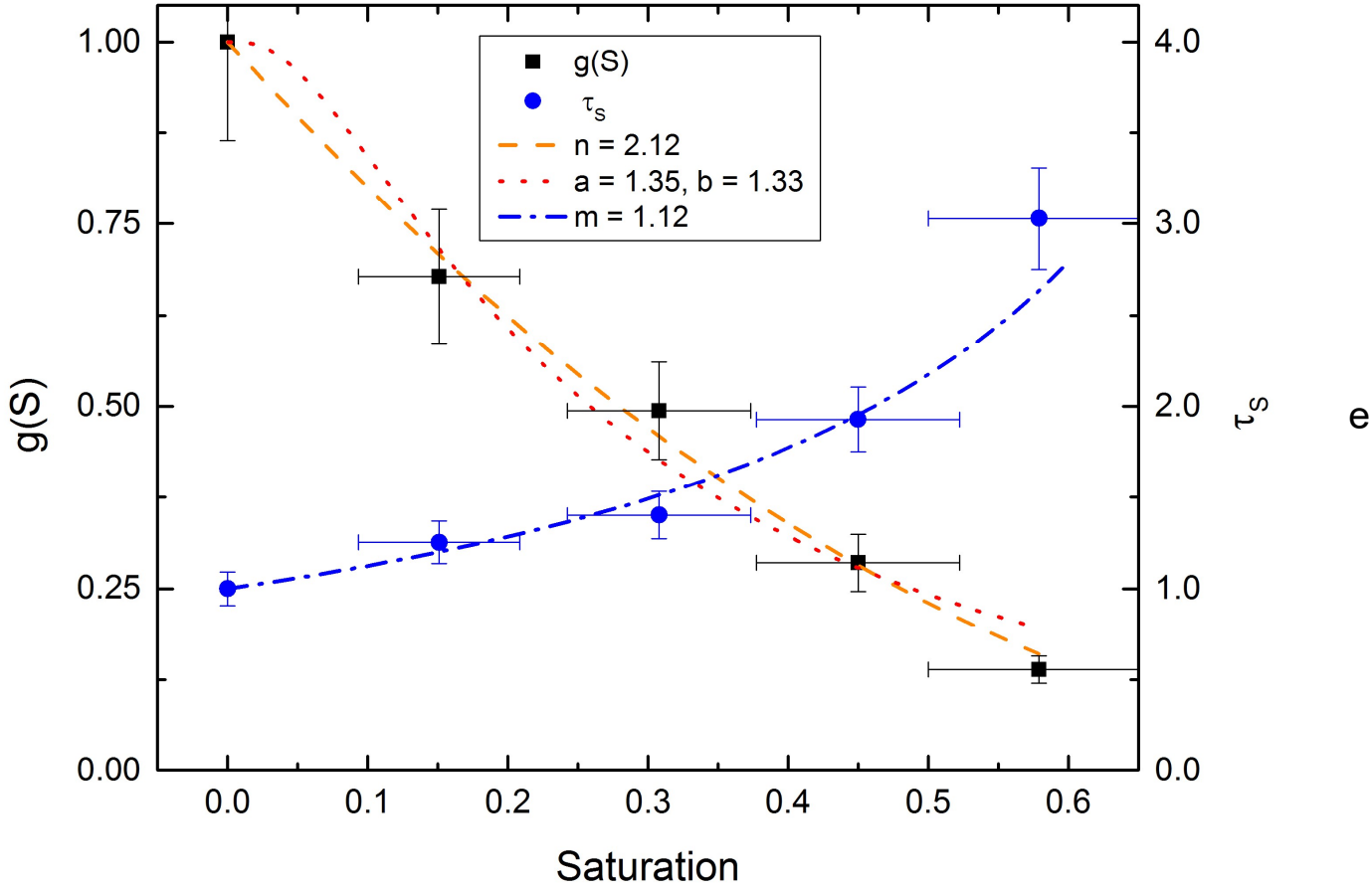


Figure (6): Results showing the relative diffusivity function, $g(S)$, fit with equations (12) and (20) and the saturation-dependent tortuosity, $\tau_{S}$, using Equation (11) with $\mathrm{m}=1-\mathrm{n}$.

A summary of the fitted parameters for all the results is also shown in Table (2).

\begin{tabular}{|l|l|l|l|l|}
\hline Material & PTFE (wt-\%) & $\mathbf{n}$ & $\mathbf{a}$ & $\mathbf{b}$ \\
\hline SGL 10 BA & 5.0 & 2.05 & 1.31 & 1.21 \\
\hline SGL 34 BA & 5.0 & 2.03 & 1.30 & 1.21 \\
\hline Toray 090 & 0.0 & 2.43 & 1.54 & 1.46 \\
\hline Toray 090 & 5.0 & 2.02 & 1.30 & 1.15 \\
\hline Toray 120 & 5.0 & 2.12 & 1.35 & 1.33 \\
\hline
\end{tabular}

Table (2): Fitted parameters for the GDL samples using equations (12) and (20).

Overall, there is little difference between PTFE treated samples with power-law exponents all close to 2 . The results agree with simulated results from both pore network models (PNM) [37], [38] and Lattice Boltzmann models (LBM) [39], [24] as well as theoretical predictions $[40,41]$. The exception is the sample with no PTFE added (Toray090) which has a higher exponent, signifying decreased transport with higher saturation. The observed difference between treated and untreated samples, though modest, suggests the PTFE successfully induced partial filling of pores in the more hydrophobic samples, allowing air to diffuse around the water through the interstitial space in the unfilled corners. 


\subsection{Comparison to Literature}

The results for a TP study of relative diffusivity conducted by Hwang and Weber tend to fit well with Equation (12) using an exponent of 3 for samples without any PTFE treatment [20]. However, in PTFE treated samples, Equation (20) was a better fit to their data, as it accounted for an initial region at low saturation where the normalized effective diffusivity remained close to 1 . The explanation given by them is that PTFE coating hinders liquid transport, leading to good phase separation and better gas phase conductivity. Their data show large scatter between samples coated with different amounts of PTFE. However, a significant variation is consistently observed between samples with and without PTFE, indicating that PTFE does indeed improve gas phase transport at a given saturation level. The impact of PTFE was not as substantial for the present study when comparing Toray 090 results.

Hwang and Weber's results for SGL 10 BA with 5\% PTFE treatment are included in

Figure (6) for direct comparison with the present study. Close agreement is generally found, especially for intermediate saturation. However, the IP results presented in this study have lower diffusivity at low saturation and higher diffusivity at higher saturation compared with the TP results of Hwang and Weber. The largest absolute difference between data-sets occurs at around 15\% saturation, which would be a typical operating fuel cell saturation, and so this warrants further investigation with a greater number of points in the data sets around low saturations. And ideally a better picture of the saturation distribution.

Equation (12) fits both treated and untreated materials equally well and no delayed onset of diffusion resistance was observed at low saturations. The exponent for 
Equation (12) was slightly higher for the untreated sample ( $n=2.43$ compared to $n=2.01$ ), so PTFE does seem to improve IP relative diffusivity slightly.

A possible explanation for the subtle effects of PTFE coating in the present study compared with the larger effect observed by Hwang and Weber probably stems from the anisotropic nature of the fibrous GDL, and its impact on the distribution of binder and PTFE. These additives tend to form web-like structures spanning the IP direction as shown by SEM images [4], [42]. Also, depending on the drying method the distribution of PTFE can be concentrated at the surfaces leaving the bulk of the material free of PTFE [43], [44], [45]. Modelling of the single-phase transport properties of GDLs with binder and PTFE structures has been conducted by El Hannach et al. [46]. Stochastically generated non-overlapping cylinders were used for the base structure and binder was built up from sections of the pore space next to fibrous intersections. They state that the impact of PTFE loading on IP properties is almost negligible, whereas TP properties (diffusivity and permeability) decrease by $10 \%$ to $40 \%$ depending on the initial porosity of the base structure. To our knowledge, no multi-phase simulations have been conducted that incorporate the different material properties and structures present within a PTFE treated GDL on the water distribution, but it is not difficult to accept that the anisotropic behaviour of the PTFE extends to liquid water distributions as well. The experimental results presented here and by Hwang and Weber would suggest that PTFE influences liquid transport in the TP direction, creating good phase separation, but does not influence the IP transport in the same manner, and this warrants further investigation. Garcia-Salaberri et al. highlight the importance of saturation distribution on the TP transport where bottlenecks can be created by liquid forming planar barriers parallel to the fibre direction [24]. The IP relative diffusion measured for homogenous samples with no IP 
porosity gradient and uniform saturation distribution, however, does not suffer from bottlenecks and remains with exponents around 2 for all local saturation values. A feature of the current study is the drying technique which should result in even evaporation of the liquid phase through air imbibition.

\section{Conclusion}

A new method for measuring the IP diffusivity of thin, partially saturated, porous material has been developed. Several fuel cell gas diffusion layer samples were investigated, with little difference found between data-sets, except for a slight decrease in relative diffusivity for untreated samples compared with PTFE treated ones. However, behaviour observed by other researchers investigating TP relative diffusivity for varying hydrophobic treatment levels was not found in the current data, suggesting that the treatment affects transport differently in different directions. The method was found to produce the expected relation between relative diffusivity and saturation for homogeneous three-dimensional media: a power law dependence with

exponent around 2. Where other techniques for measuring effective properties of dry material are not suitable for measuring partially saturated material, this method has proved successful. Future work could utilise the same set-up with minor modification to measure the relative permeability and also study the effect of compression and inhomogeneity on the relative transport properties. In addition the bottom plate may be modified to allow for in-situ liquid injection, to investigate the effect of differing saturation distributions.

Acknowledgements:

The first author would like to gratefully acknowledge the funding provided by the EPSRC under grant no. 1269327. 


\section{Appendix}

\subsection{Example Error Calculation in Saturation}

\begin{tabular}{|c|c|c|c|}
\hline Quantity & Value & $\Delta+/=$ & Uncertainty Calculation \\
\hline Length $(\mathrm{I})[\mathrm{m}]$ & $7 e-2$ & $1 e-3$ & Visual Estimate \\
\hline Width $(\mathrm{w})[\mathrm{m}]$ & $1 \mathrm{e}-2$ & $5 e-4$ & Visual Estimate \\
\hline Height (h) [m] & $2.84 \mathrm{e}-4$ & $1.2 \mathrm{e}-5$ & $\begin{array}{l}\text { Measured variance, } \sigma \text {, from } 10 \text { points along } \\
\text { sample length with micrometer }\end{array}$ \\
\hline $\begin{array}{l}\text { Height } \\
\text { Compressed } \\
\text { (hc) }[\mathrm{m}]\end{array}$ & $2.54 \mathrm{e}-4$ & $1 e-5$ & Shim tolerance estimate \\
\hline $\begin{array}{l}\text { Volume (V) } \\
{\left[\mathrm{cm}^{3}\right]} \\
\text { Compressed } \\
\left(\mathrm{V}_{\mathrm{C}}\right)\end{array}$ & $\begin{array}{l}0.1988 \\
0.1778\end{array}$ & $\begin{array}{l}1.33 \mathrm{e}-2 \\
1.16 \mathrm{e}-2\end{array}$ & 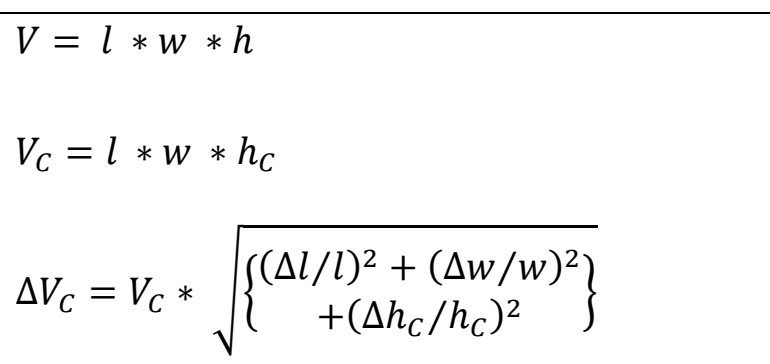 \\
\hline Porosity $(\phi)$ & 0.827 & $8 e-3$ & [29] \\
\hline $\begin{array}{l}\text { Porosity } \\
\text { compressed } \\
\left(\phi_{C}\right)\end{array}$ & 0.81 & $5 \mathrm{e}-2$ & $\begin{aligned} \phi_{C} & =1-\left(\left(h / h_{C}\right) *(1-\phi)\right) \\
\Delta \phi_{C} & \left.=\phi_{C} * \sqrt{\left\{\begin{array}{c}(\Delta h / h)^{2}+\left(\Delta h_{C} / h_{C}\right)^{2} \\
+(\Delta \phi / \phi)^{2}\end{array}\right.}\right\}\end{aligned}$ \\
\hline
\end{tabular}




\begin{tabular}{|l|l|l|l|}
\hline $\begin{array}{l}\text { Dry weight } \\
\left(\mathrm{M}_{\text {dry }} \text { [g] }\right.\end{array}$ & 0.0625 & $5 \mathrm{e}-4$ & Estimate from mass balance \\
\hline $\begin{array}{l}\text { Wet weight } \\
\left(\mathrm{M}_{\text {wet }} \text { [g] }\right.\end{array}$ & 0.150 & $5 \mathrm{e}-3$ & Estimate loss from evaporation \\
\hline $\begin{array}{l}\text { Saturation } \\
\text { (S) }\end{array}$ & 0.61 & 0.06 & $S=\left(M_{\text {wet }}-M_{d r y}\right) /\left(V_{c} * \phi_{C} * \rho_{\text {water }}\right)$ \\
\end{tabular}

\section{References}

[1] K. Jiao, X. Li, Water transport in polymer electrolyte membrane fuel cells, Prog. Energy Combust. Sci. 37 (2011) 221-291.

doi:10.1016/j.pecs.2010.06.002.

[2] D. Baker, C. Wieser, K. Neyerlin, M. Murphy, The use of limiting current to determine transport resistance in PEM fuel cells, ECS Trans. 3 (2006) 989999. doi:10.1149/1.2356218.

[3] U. Beuscher, Experimental Method to Determine the Mass Transport Resistance of a Polymer Electrolyte Fuel Cell, J. Electrochem. Soc. 153 (2006) A1788. doi:10.1149/1.2218760.

[4] R. Flückiger, S.A. Freunberger, D. Kramer, A. Wokaun, G.G. Scherer, F.N. Büchi, Anisotropic, effective diffusivity of porous gas diffusion layer materials for PEFC, Electrochim. Acta. 54 (2008) 551-559.

doi:10.1016/j.electacta.2008.07.034.

[5] D. Kramer, S.A. Freunberger, R. Flückiger, I.A. Schneider, A. Wokaun, F.N. Büchi, G.G. Scherer, Electrochemical diffusimetry of fuel cell gas diffusion layers, J. Electroanal. Chem. 612 (2008) 63-77. doi:10.1016/j.jelechem.2007.09.014.

[6] C. Quick, D. Ritzinger, W. Lehnert, C. Hartnig, Characterization of water transport in gas diffusion media, J. Power Sources. 190 (2009) 110-120. doi:10.1016/j.jpowsour.2008.07.093.

[7] T. Hottinen, O. Himanen, S. Karvonen, I. Nitta, Inhomogeneous compression of PEMFC gas diffusion layer, J. Power Sources. 171 (2007) 113-121. doi:10.1016/j.jpowsour.2006.10.076.

[8] L.M. Pant, S.K. Mitra, M. Secanell, Absolute permeability and Knudsen 
diffusivity measurements in PEMFC gas diffusion layers and micro porous layers, J. Power Sources. 206 (2012) 153-160.

doi:10.1016/j.jpowsour.2012.01.099.

[9] P. Mangal, L.M. Pant, N. Carrigy, M. Dumontier, V. Zingan, S. Mitra, M. Secanell, Experimental study of mass transport in PEMFCs: Through plane permeability and molecular diffusivity in GDLs, Electrochim. Acta. 167 (2015) 160-171. doi:10.1016/j.electacta.2015.03.100.

[10] I. Nitta, S. Karvonen, O. Himanen, M. Mikkola, Modelling the Effect of Inhomogeneous Compression of GDL on Local Transport Phenomena in a PEM Fuel Cell, Fuel Cells. 8 (2008) 410-421. doi:10.1002/fuce.200700058.

[11] P.J. Hamilton, B.G. Pollet, Polymer Electrolyte Membrane Fuel Cell (PEMFC) Flow Field Plate: Design, Materials and Characterisation, Fuel Cells. 10 (2010) 489-509. doi:10.1002/fuce.201000033.

[12] M.S. Ismail, D.B. Ingham, K.J. Hughes, L. Ma, M. Pourkashanian, Effective diffusivity of polymer electrolyte fuel cell gas diffusion layers: An overview and numerical study, Int. J. Hydrogen Energy. 40 (2015) 10994-11010. doi:10.1016/j.jjhydene.2015.06.073.

[13] M. Ciureanu, R. Roberge, Electrochemical impedance study of PEM fuel cells. Experimental diagnostics and modeling of air cathodes, J. Phys. Chem. B. 105 (2001) 3531-3539. doi:10.1021/jp003273p.

[14] T.J. Mason, J. Millichamp, P.R. Shearing, D.J.L. Brett, A study of the effect of compression on the performance of polymer electrolyte fuel cells using electrochemical impedance spectroscopy and dimensional change analysis, Int. J. Hydrogen Energy. 38 (2013) 7414-7422. doi:10.1016/j.jjhydene.2013.04.021.

[15] D.R. Baker, D.A. Caulk, K.C. Neyerlin, M.W. Murphy, Measurement of Oxygen Transport Resistance in PEM Fuel Cells by Limiting Current Methods, J. Electrochem. Soc. 156 (2009) B991. doi:10.1149/1.3152226.

[16] S. Chevalier, J. Lee, N. Ge, R. Yip, P. Antonacci, Y. Tabuchi, T. Kotaka, In operando measurements of liquid water saturation distributions and effective diffusivities of polymer electrolyte membrane fuel cell gas diffusion layers, Electrochim. Acta. 210 (2016) 792-803. doi:10.1016/j.electacta.2016.05.180.

[17] J.H. Rohling, J. Shen, C. Wang, J. Zhou, C.E. Gu, Determination of binary diffusion coefficients of gases using photothermal deflection technique, Appl. Phys. B Lasers Opt. 87 (2007) 355-362. doi:10.1007/s00340-007-2595-9.

[18] N. Astrath, J. Shen, D. Song, The effect of relative humidity on binary gas diffusion, J. Phys. Chem. B. (2009) 8369-8374. doi:10.1021/jp900796w.

[19] R. Rashapov, F. Imami, J.T. Gostick, A method for measuring in-plane effective diffusivity in thin porous media, Int. J. Heat Mass Transf. 85 (2015) 367-374. doi:10.1016/j.ijheatmasstransfer.2015.01.101.

[20] G.S. Hwang, A.Z. Weber, Effective-Diffusivity Measurement of PartiallySaturated Fuel-Cell Gas-Diffusion Layers, J. Electrochem. Soc. 159 (2012) F683-F692. doi:10.1149/2.024211jes. 
[21] Y. Utaka, I. Hirose, Y. Tasaki, Characteristics of oxygen diffusivity and water distribution by $\mathrm{X}$-ray radiography in microporous media in alternate porous layers of different wettability for moisture control in gas diffusion layer of PEFC, Int. J. Hydrogen Energy. 36 (2011) 9128-9138.

doi:10.1016/j.jihydene.2011.04.152.

[22] R. Koresawa, Y. Utaka, Precise measurement of effective oxygen diffusivity for microporous media containing moisture by review of galvanic cell oxygen absorber configuration, Int. J. Heat Mass Transf. 76 (2014) 549-558. doi:10.1016/j.ijheatmasstransfer.2014.05.005.

[23] J. Becker, R. Flückiger, M. Reum, F.N. Büchi, F. Marone, M. Stampanoni, Determination of Material Properties of Gas Diffusion Layers: Experiments and Simulations Using Phase Contrast Tomographic Microscopy, J. Electrochem. Soc. 156 (2009) B1175. doi:10.1149/1.3176876.

[24] P.A. García-Salaberri, G. Hwang, M. Vera, A.Z. Weber, J.T. Gostick, Effective diffusivity in partially-saturated carbon-fiber gas diffusion layers: Effect of through-plane saturation distribution, Int. J. Heat Mass Transf. 86 (2015) 319 333. doi:10.1016/j.ijheatmasstransfer.2015.02.073.

[25] P.A. García-Salaberri, J.T. Gostick, G. Hwang, A.Z. Weber, M. Vera, Effective diffusivity in partially-saturated carbon-fiber gas diffusion layers: Effect of local saturation and application to macroscopic continuum models, J. Power Sources. 296 (2015) 440-453. doi:10.1016/j.jpowsour.2015.07.034.

[26] L. Shen, Z. Chen, Critical review of the impact of tortuosity on diffusion, Chem. Eng. Sci. 62 (2007) 3748-3755. doi:10.1016/j.ces.2007.03.041.

[27] N. Epstein, On tortuosity and the tortuosity factor in flow and diffusion through porous media, Chem. Eng. Sci. 44 (1989) 777-779. doi:10.1016/00092509(89)85053-5.

[28] R.R. Rashapov, J.T. Gostick, In-Plane Effective Diffusivity in PEMFC Gas Diffusion Layers, Transp. Porous Media. (2016). doi:10.1007/s11242-0160648-4.

[29] R.R. Rashapov, J. Unno, J.T. Gostick, Characterization of PEMFC Gas Diffusion Layer Porosity, J. Electrochem. Soc. 162 (2015) F603-F612. doi:10.1149/2.0921506jes.

[30] J.T. Gostick, M.A. loannidis, W. Fowler, M.D. Pritzker, Characterization of the Capillary Properties of Gas Diffusion Media, Model. Diagnostics Polym. Electrolyte Fuel Cells Mod. Asp. Electrochem. (2010) 225-254. doi:10.1007/978-0-387-98068-3.

[31] J. Crank, The mathematics of diffusion, Oxford university press, 1979.

[32] J.E. Guyer, D. Wheeler, J.A. Warren, FiPy: Partial Differential Equations with Python, Comput. Sci. Eng. 11 (2009) 6-15. doi:10.1109/MCSE.2009.52.

[33] E. Jones, T. Oliphant, P. Peterson, SciPy: Open source scientific tools for Python, (2001). http://www.scipy.org/.

[34] N. Zamel, N.G.C. Astrath, X. Li, J. Shen, J. Zhou, F.B.G. Astrath, H. Wang, Z.S. Liu, Experimental measurements of effective diffusion coefficient of oxygen- 
nitrogen mixture in PEM fuel cell diffusion media, Chem. Eng. Sci. 65 (2010) 931-937. doi:10.1016/j.ces.2009.09.044.

[35] Y. Sun, I.S. Wichman, On transient heat conduction in a one-dimensional composite slab, Int. J. Heat Mass Transf. 47 (2004) 1555-1559.

doi:10.1016/j.ijheatmasstransfer.2003.09.011.

[36] M.S. P. Mangal, M. Dumontier, N. Carrigy, Measurements of permeability and effective in-plane gas diffusivity of gas diffusion media under compression, ECS Trans. 64 (2014) 487-499.

[37] J.H. Nam, M. Kaviany, Effective diffusivity and water-saturation distribution in single- and two-layer PEMFC diffusion medium, Int. J. Heat Mass Transf. 46 (2003) 4595-4611. doi:10.1016/S0017-9310(03)00305-3.

[38] J.T. Gostick, Random Pore Network Modeling of Fibrous PEMFC Gas Diffusion Media Using Voronoi and Delaunay Tessellations, J. Electrochem. Soc. 160 (2013) F731-F743. doi:10.1149/2.009308jes.

[39] T. Rosen, J. Eller, J. Kang, N.I. Prasianakis, J. Mantzaras, F.N. Buchi, Saturation Dependent Effective Transport Properties of PEFC Gas Diffusion Layers, J. Electrochem. Soc. 159 (2012) F536-F544. doi:10.1149/2.005209jes.

[40] M. Sahimi, B.D. Hughes, L.E. Scriven, H.T. Davis, Critical exponent of percolation conductivity by finite- size scaling, J. Phys. C Solid State Phys. 16 (1983) L521-L527. doi:10.1088/0022-3719/16/16/004.

[41] R.B. Pandey, D. Stauffer, A. Margolina, J.G. Zabolitzky, Diffusion on random systems above, below, and at their percolation threshold in two and three dimensions, J. Stat. Phys. 34 (1984) 427-450. doi:10.1007/BF01018553.

[42] N. Zamel, X. Li, J. Shen, Correlation for the Effective Gas Diffusion Coefficient in Carbon Paper Diffusion Media, Energy \& Fuels. 23 (2009) 6070-6078. doi:10.1021/ef900653x.

[43] N. Khajeh-Hosseini-Dalasm, T. Sasabe, T. Tokumasu, U. Pasaogullari, Effects of polytetrafluoroethylene treatment and compression on gas diffusion layer microstructure using high-resolution X-ray computed tomography, J. Power Sources. 266 (2014) 213-221. doi:10.1016/j.jpowsour.2014.05.004.

[44] T. Sasabe, G. Inoue, S. Tsushima, S. Hirai, T. Tokumasu, U. Pasaogullaria, Investigation on Effect of PTFE Treatment on GDL Micro-structure by Highresolution X-ray CT, ECS Trans. 50 (2012) 735-744. doi:10.1149/05002.0735ecst.

[45] A. Rofaiel, J.S. Ellis, P.R. Challa, A. Bazylak, Heterogeneous through-plane distributions of polytetrafluoroethylene in polymer electrolyte membrane fuel cell gas diffusion layers, J. Power Sources. 201 (2012) 219-225. doi:10.1016/j.jpowsour.2011.11.005.

[46] M. El Hannach, E. Kjeang, Stochastic Microstructural Modeling of PEFC Gas Diffusion Media, J. Electrochem. Soc. 161 (2014) F951-F960. doi:10.1149/2.1141409jes. 